\title{
Prediction Model of Vibration Feature for Equipment Maintenance Based on Full Vector Spectrum
}

\author{
Lei Chen, ${ }^{1,2}$ Jie Han, ${ }^{1}$ Wenping Lei, ${ }^{1}$ ZhenHong Guan, ${ }^{1}$ and Yajuan Gao ${ }^{1}$ \\ ${ }^{1}$ Institute of Vibration Engineering, Zhengzhou University, Zhengzhou 450001, China \\ ${ }^{2}$ School of Chemical Engineering and Energy, Zhengzhou University, Zhengzhou 450001, China \\ Correspondence should be addressed to Lei Chen; chenlei@zzu.edu.cn
}

Received 6 October 2016; Revised 6 February 2017; Accepted 21 February 2017; Published 12 March 2017

Academic Editor: Hassan Askari

Copyright (c) 2017 Lei Chen et al. This is an open access article distributed under the Creative Commons Attribution License, which permits unrestricted use, distribution, and reproduction in any medium, provided the original work is properly cited.

\begin{abstract}
Establishing a prediction model is a key step for the implementation of prognostic and health management. The prediction model can be used to forecast the change trend of the characteristics of the vibration signal and analyze the potential failure in the future. Taking the vibration of power plant steam turbine as an example, the full vector fusion and fault prediction were studied. Due to the fact that the evaluation of the machine fault with only one transducer may result in a fault judgement with partiality, an information fusion method based on the theory of full vector spectrum was adopted to extract the vibration feature. An autoregressive prediction model was established. The collected vibration signals with pairing channels were fused. The time sequence of the fused vectors and spectrums were used to build the prediction model. The amplitude of main vector of rotating frequency and spectrum order structure were analyzed and predicted. The uncertainty of the spectrum structure can be eliminated by the information fusion. The reliability of the fault prediction was improved. The study on vibration prediction model system laid a technical foundation for the fault prognostic research.
\end{abstract}

\section{Introduction}

By the techniques of prediction, equipment status deterioration laws can be drawn out from the machine historical state parameters and thus potential faults can be predicted. The maintenance plan can be arranged reasonably to ensure the safe operation of the equipment [1]. Prediction modeling and vibration data feature extraction are the key predictive maintenance technologies. In recent years, scholars have conducted lots of work on the aspect of equipment fault prediction, and many prediction methods are proposed [2-6]. These methods can be roughly divided into three categories: knowledge based, model based, and data based. Some expert systems and fuzzy logic [7-9] are the method based on knowledge. This method can make use of expert knowledge and experience, and it does not need to be very accurate model. But the acquisition of the knowledge is quite difficult. The method of filter prediction and the method of modeling based on the fault mechanism are the methods based on model [10-12]. When the mathematical model of the diagnosis object is accurate, this kind of method can get the accurate result of fault prediction. However, it is difficult to establish accurate mathematical model for the complex dynamic system. So this method is very limited and expensive. The prediction methods of autoregressive forecast, grey theory, neural network, and so forth are the methods based on data $[4-6,13-16]$. By mining the implicit information from the industrial field data, this type of method has a wide range of engineering application. The prediction method based on data has a wide range and low cost. It is the most practical method and has become the research focus and development trend in the field of fault diagnosis and prediction. In this paper, an autoregressive forecasting model is established based on historical vibration data.

When a fault prediction model was established, fault signature was obtained from vibration transducers. Although multiple transducers were used in the monitoring system, fault signature was still extracted from one of them. Based on the change of eigenvalues or characteristic vector, possible failures could be predicted. According to the theory of full spectrum and holographic and full vector spectrum $[1,17-19]$, the vibration track under a vibration harmonic is an ellipse. 


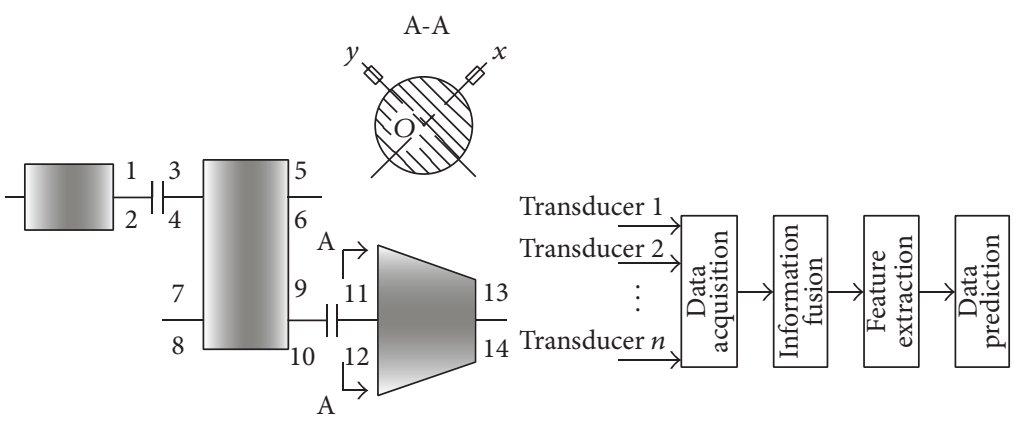

FIGURE 1: Diagram of prediction system based on full vector spectrum.

The vibration obtained by a transducer is the projection of the vibration trace in the transducer's installation direction. The measurement results have certain limitation and onesidedness, and even in some cases different conclusions will be drawn according to the transducers installed in different directions. So in this study signal vector was extracted based on the theory of full vector spectrum in the process of modeling to overcome the partial impact. The equipment vibration vector prediction model was established to predict degradation trend and analyze the possibility of equipment failure.

\section{Structure of Full Vector Prediction}

The structure of the full vector fault prediction and diagnosis system is shown in Figure 1. The Arabic numerals $1 \sim 14$ is the measuring points. In the same interface, two transducers are installed in $X-Y$ or vertical layout. For example, in the A-A section, two transducers are installed in $X$ and $Y$ direction to acquire the rotor vibration. The data acquisition system transforms the analog vibration into digital data after signal conditioning, filter, and analog to digital conversion. The equipment running status can be evaluated and possible failure can be analyzed according to the result of prediction. When the rotor is running, the orbit of shaft center on each harmonic is an ellipse. Under each harmonic, the signal obtained by the transducer is the projection of the vibration trace in the corresponding direction. The overall signal measured by a transducer is the superposition of projections of all measurable frequencies. So signals of a single transducer cannot reflect the vibration of the equipment comprehensively and information fusion is necessary. In this study, the difference of spectrums is addressed between signals obtained by a signal transducer and signals after information fusion. The time sequence vibration vector is used as input parameters and a data driven AR prediction model is built with fixed order. The prediction model's coefficient was updated with the changing of the vibration signal to improve the model with the ability of selfcorrecting.

\section{Feature Extraction and Prediction}

Since, in the same measurement section, vibration obtained by a single transducer has certain one-sidedness, information fusion is needed to extract the vibration vector. The vibration vector is the fused signal of the two transducers installed in different directions. It can reflect the actual circumstances of the machine vibration. It is a feasible way to judge the trend of equipment operating status by vibration vector.

3.1. Extract of Vibration Vector. Assuming that the two transducers of $x$ and $y$ direction were installed in section A-A of Figure 1 to measure the rotor vibration, vibrations acquired by the two transducers of a steady rotating machinery are a combination of motion orbits for some harmonic waves $w_{i}(i=1,2, \ldots)$. For a certain harmonic $i$, assume the following definitions: $X_{i}$ stands for the amplitude of vibration signal in the direction $x ; Y_{i}$ stands for the amplitude of vibration signal of the harmonic $i$ in the direction $y ; \emptyset_{x i}$ stands for the phase angle of the harmonic $i$ in the direction $x ; \emptyset_{y i}$ stands for the phase angle of the harmonic $i$ in the direction $y ; X_{c i}$ stands for the amplitude of vibration in direction $x$ of harmonic $i$ when $t=0 ; Y_{c i}$ stands for the amplitude of vibration in direction $y$ of harmonic $i$ when $t=0 ; X_{s i}$ and $Y_{s i}$ have no physical meaning and were used for the geometry calculation of harmonic track. Then there are the following equations:

$$
\begin{aligned}
& X_{c i}=X_{i} \cos \emptyset_{x i} \\
& X_{s i}=X_{i} \sin \emptyset_{x i} \\
& Y_{c i}=Y_{i} \cos \emptyset_{y i} \\
& Y_{s i}=Y_{i} \sin \emptyset_{y i} .
\end{aligned}
$$

The motion equations in $X$ and $Y$ direction can be generally expressed as

$$
\begin{aligned}
x & =\sum_{i=1}^{\infty} X_{i} \cos \left(\omega_{i} t+\emptyset_{x i}\right)=\operatorname{Re}\left(\sum_{i=1}^{\infty} \bar{X}_{i} e^{j \omega_{i} t}\right) \\
& =\operatorname{Re}\left[\sum_{i=1}^{\infty}\left(x_{c i}+j x_{s i}\right) e^{j \omega_{i} t}\right] \\
y & =\sum_{i=1}^{\infty} Y_{i} \cos \left(\omega_{i} t+\emptyset_{y i}\right)=\operatorname{Re}\left(\sum_{i=1}^{\infty} \bar{Y}_{i} e^{j \omega_{i} t}\right) \\
& =\operatorname{Re}\left[\sum_{i=1}^{\infty}\left(y_{c i}+j y_{s i}\right) e^{j \omega_{i} t}\right] .
\end{aligned}
$$




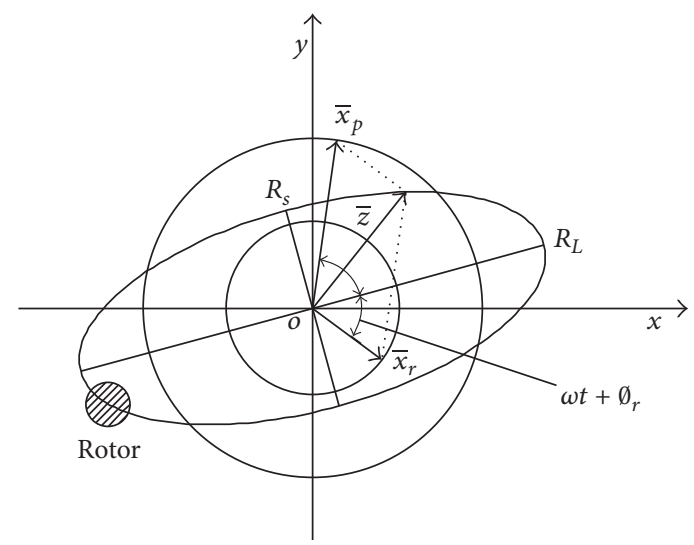

FIGURE 2: Ellipse orbit.

After some trigonometric function calculations, (3) can be reached. The equation indicates that the vibration trajectory is an ellipse in every harmonic $i(i=1,2,3, \ldots)$.

$$
\begin{aligned}
\left(Y_{c i}^{2}\right. & \left.+Y_{s i}^{2}\right) x_{i}^{2}+\left(X_{c i}^{2}+X_{s i}^{2}\right) y_{i}^{2} \\
& -2\left(X_{c i} Y_{s i}+X_{s i} Y_{c i}\right) x_{i} y_{i}=\left(X_{s i} Y_{s i}-X_{c i} Y_{c i}\right)^{2} .
\end{aligned}
$$

As shown in Figure 2, $R_{L}$ is the half major axis of the ellipse. $R_{S}$ is the half minor axis. Elliptic trajectory in kinematics can be regarded as the synthesis of two circular motions of the same frequency and opposite direction $[1,17$, 18]. There exists the following equation.

$$
\bar{z}=\bar{x}_{p}+\bar{x}_{r}
$$

In Figure $2, \bar{z}$ represents a point on the rotor trajectory at a certain time. $\bar{x}_{p}$ is the forward precession circle vector with phase angle $\emptyset_{p} \cdot \bar{x}_{r}$ is the backward precession circle vector with phase angle $\emptyset_{r}$.

If the ellipse was drawn in the same coordinate system, the running state of the rotor can be understood directly [18]. Industry wide of a single transducer to assess dynamics has been replaced by use of two or more transducers and key-phasor as fully described in detail in the classic works by Dr. Donald Bently [17]. Dr. Bently's classic work methodically and accurately explains in great detail the use of diagnostic equipment, theory, and applications. Theory of full spectrum proposed by Dr. Bently considers that the radius of the forward circle and backward circle which form the harmonic ellipse can be displayed, respectively, according to the frequency axis of positive and negative. By comparing the amplitudes of the harmonic under positive and negative frequencies, the direction of precession of the ellipse and vibration intensity can be determined.

The maximum displacement of the rotor is at the vertex point of the major axis of the ellipse. The major axis of the ellipse is the biggest displacement of the vibration. It can stand for the size of the rotor vibration. Here the ellipse's long half axis was called the main vibration vector, and the short half axis was called the assistant vibration vector.

According to the theory of full vector spectrum, the main vector of vibration harmonic can be simplified as a simple calculation by FFT $[1,2]$. Assuming that $x_{i}$ and $y_{i}$ are the signals acquired by the transducers $x$ and $y$. Here $i=0,1,2, \ldots, N-1, N$ are the sampling points. A complex sequence $z_{i}$ can be constituted by the $x_{i}$ and $y_{i}$; that is, $z_{i}=$ $x_{i}+j y_{i} . Z_{k}$ can be obtained by Fourier transform of $z_{i}$ data series; here $k=1,2, \ldots, N / 2-1$. After a series of calculations, (5) can be obtained.

$$
\begin{aligned}
& R_{L k}=\frac{1}{2 N}\left[\left|Z_{k}\right|+\left|Z_{N-k}\right|\right] \\
& R_{S k}=\frac{1}{2 N}\left[\left|Z_{k}\right|-\left|Z_{N-k}\right|\right] \\
& \qquad\left(k=0,1,2, \ldots, \frac{N}{2}-1\right) .
\end{aligned}
$$

In (5), $R_{L k}$ and $R_{S k}$ are the main and assistant vibration vectors. Its spectral structure can reflect the operation state of the machine comprehensively.

3.2. Autoregressive Prediction Model. Prediction method of vibration vector was based on autoregressive (AR) model. Autoregressive model is a common form of time series [20]. It is a special case of Autoregressive Moving Average (ARMA) model.

For a stationary time sequence $\left\{x_{t}\right\}$, if the value of $x_{t}$ is concerned not only with the data $x_{t-1}, x_{t-2}, \ldots, x_{t-n}(n=$ $1,2, \ldots, n)$ but also with the disturbance $\alpha_{t-1}, \alpha_{t-2}, \alpha_{t-m}(m=$ $1,2, \ldots, m)$, the general structure of $\operatorname{ARMA}(n, m)$ according to the idea of multiple linear regression is as follows:

$$
x_{t}=\sum_{i=1}^{n} \varphi_{i} x_{t-i}-\sum_{j=1}^{m} \theta_{j} \alpha_{t-j}+\alpha_{t} .
$$

Here $n$ stands for the order of $\operatorname{AR}(n) ; m$ stands for the order of $\mathrm{MA}(m) ; \varphi_{i}(i=1,2, \ldots, n)$ stands for the parameters of autoregressive; $\theta_{j}(j=1,2, \ldots, m)$ stands for the parameters of moving average; $\alpha_{t}$ stands for a sequence of independent and identically distributed random variables. The mean of $\alpha_{t}: E\left(\alpha_{t}\right)=0$; the variance of $\alpha_{t}: \operatorname{Var}\left(\alpha_{t}\right)=\sigma_{\varepsilon}^{2}>$ 0 .

When $\theta_{j}=0$, the $\operatorname{ARMA}(n, m)$ model has no moving average. At this circumstance, the model was called autoregressive model with order $n$, that is, $\operatorname{AR}(n)$. Its structure is as follows:

$$
x_{t}=\sum_{i=1}^{n} \varphi_{i} x_{t-i}+\alpha_{t}
$$

The parameter estimation of AR model is linear estimation, which is fast and robust, and it is easy to be used in practical engineering. $\operatorname{ARMA}(n, m)$ model is equivalent to the $\operatorname{AR}(n)$ model in the case of large order, so the fault prediction can be carried out using $\operatorname{AR}(n)$ model.

The establishment of the model is shown in Figure 3. The first step is to identify the model, that is, to determine whether the vibration vector data sequence can be used for the AR prediction. The basic condition of the AR model is that the time sequence data must meet the requirement of stability. Whether the data is stable can be determined by the changes of self-correlation coefficient $\rho_{j}$ with $j[21,22]$. If $\rho_{j}$ approach 


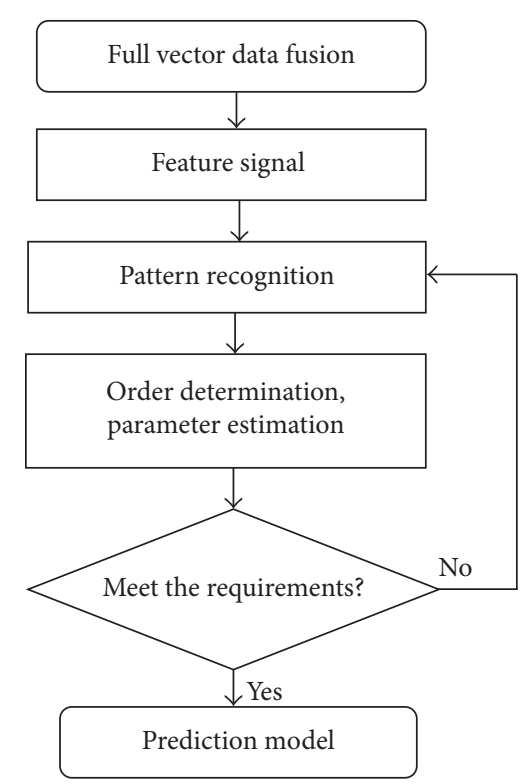

FIGURE 3: Process of prediction model establishment.

rapidly zero with the increase of $j$, the time series data meets the requirement of stability. On the contrary, it does not meet the requirement of stability. For the nonstationary data, advanced stationary processing is needed.

The order of the model is an important factor that affects the accuracy of the prediction results. Once the order number is selected, the prediction model structure is determined. At present, the commonly used order selection criteria were information criterion [23-25] and the empirical criteria given by the field experts [26]. In the literature [27], some empirical criterion for selecting the model order under different data number of time series was proposed. The selection of the order also needs to combine the experimental analysis with the current data.

For the $\operatorname{AR}(n)$ model, the parameters needing estimation were the mean $\mu$, variance $\sigma_{\alpha}^{2}$, and the coefficients $\varphi=$ $\left[\varphi_{1}, \varphi_{2}, \ldots, \varphi_{p}\right]$.

Mean value was estimated by

$$
\mu=\bar{X}=\sum_{i=1}^{n} \frac{X_{i}}{n} .
$$

Self-covariance was estimated by

$$
\widehat{\gamma_{k}}=\frac{1}{n} \sum_{i=1}^{n-k}\left(Z_{i}-\bar{Z}\right)\left(Z_{i+k}-\bar{Z}\right) .
$$

For the sample $\left\{\widetilde{X_{t}}\right\}$, if the self-covariance $\gamma_{k}$ is known, the solution can be obtained by the Yule-Waller estimation equation. That is,

$$
\left[\begin{array}{c}
\gamma_{1} \\
\gamma_{2} \\
\vdots \\
\gamma_{n}
\end{array}\right]=\left[\begin{array}{cccc}
\gamma_{0} & \gamma_{1} & \cdots & \gamma_{n-1} \\
\gamma_{1} & \gamma_{2} & \cdots & \gamma_{n-2} \\
\vdots & \vdots & & \vdots \\
\gamma_{n-1} & \gamma_{n-2} & \cdots & \gamma_{0}
\end{array}\right]\left[\begin{array}{c}
\varphi_{1} \\
\varphi_{2} \\
\vdots \\
\varphi_{n}
\end{array}\right] .
$$

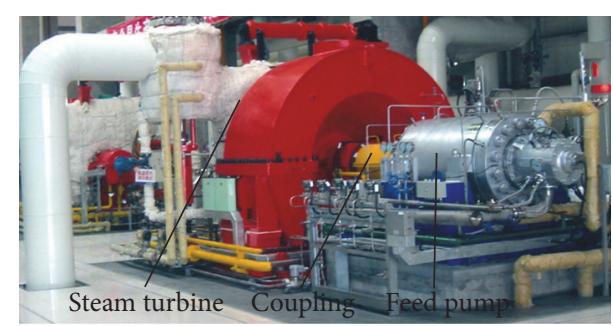

FIgURE 4: Photo of the steam turbine feed pump.

Replace $\gamma_{k}$ of the formula (10) with $\widehat{\gamma_{k}}$; the parameter estimates $\widehat{\varphi}=\left[\widehat{\varphi}_{1}, \widehat{\varphi}_{2}, \ldots, \widehat{\varphi}_{n}\right]$ will be obtained. After determining the order of the AR model and the coefficients of each order, the prediction model is established. Statistical value test can be used to determine whether the model can be used for prediction.

3.3. Application of Prediction Model. The AR prediction model is established according to the historical data. In the process of using, as the data is updated, the number of order of the AR model remains unchanged, but the coefficients of each order are revised. A relational database based on Microsoft SQL Server was built in the monitoring system for the purpose of facilitating the trend analysis and fault prediction. There are real-time data tables and historical data tables in the database. Every two seconds the acquisition system collects a set of data for real-time monitoring. The prediction system reads the data from the historical data table and sends it into the established AR model for the trend analysis and numerical prediction. Historical data is divided into seven levels. The first level stores the data of the last 8 hours; the second level stores the data of the last day; the third level stores the data of the last week; the fourth level stores the data of the last month; the fifth level stores the data of the last three months; the sixth level stores the data for the last six months; and the seventh level stores the data for the last year. Historical data older than one year is no longer used for fault prediction. When the number of predicted steps was determined, the time span of prediction results was different with the different time data series.

\section{Test and Experiment}

4.1. Comparison of Full Vector Spectrum and Traditional Spectrum. In order to verify the correctness of the method for data fusion and prediction, an online monitoring system was installed in a power plant in China, and the vibration data were collected. The steam turbine of the power plant drives the feed water pump to supply water for the boiler. The rotor structure of the steam turbine feed pump group is composed of a steam turbine rotor, a feed water pump shaft, and a flexible coupling which is used for connecting the rotor and the feed pump shaft. The steam turbine feed pump on-site photo is shown in Figure 4. Figure 5 is the internal structure. Vibration measuring points are shown in Figure 6. In the figure, S1 S10 are eddy current transducers. S1 S8 were used to measure the rotor radial displacement; S9 was used to measure the axial position of the rotor; S10 


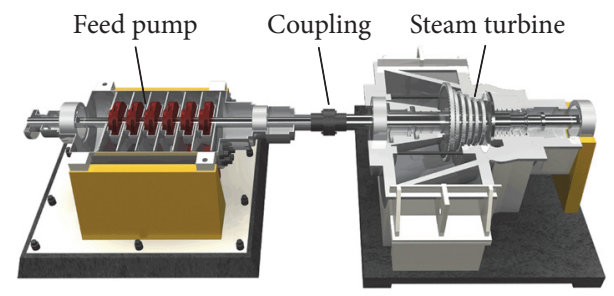

FIGURE 5: Internal structure of the steam turbine feed pump.

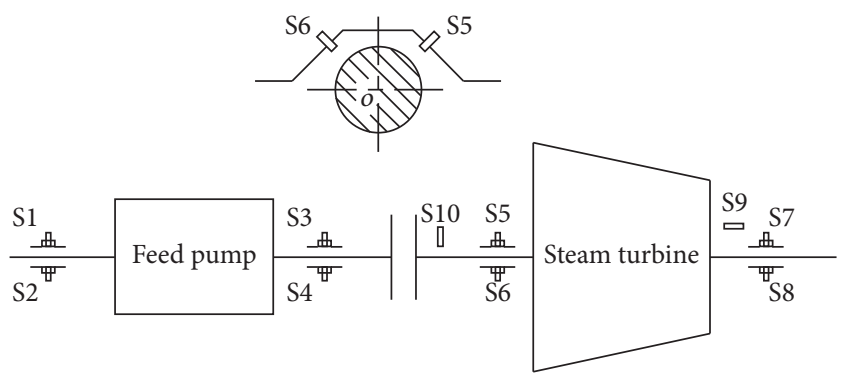

FIGURE 6: Layout of vibration measuring point.

was used to measure the rotating speed and provide the key phase signal. S5 and S6 are eddy current transducers installed perpendicular to each other, which are used to monitor the radial vibration of the rotor at the output end of the turbine. S5 was installed in the $x$ direction. S6 was installed in the $y$ direction.

The sampling frequency is $1600 \mathrm{Sa} / \mathrm{s}$; sampling length is 1024 points; the rotor speed is $3000 \mathrm{r} / \mathrm{min}$. The vibration waveforms of S5 and S6 are shown in Figure 7. In time domain, the waveforms indicate that the overall vibration amplitude of $X$ and $Y$ direction is of considerable size. In frequency domain as shown in Figure 8, the frequency structure of $x$ and $y$ direction is quite different. According to the spectrum of $x$ direction, it seems that the vibration amplitude mainly relates to the double frequency, and perhaps there exists fault of misalignment; however, after observing the spectrum in $y$ direction, it seems that the vibration relates to the rotating frequency. Therefore, the fault may be related to the unbalance. Different diagnosis conclusions may be drawn on the machine by signals of different directions on the same rotor interface. Making a diagnosis conclusion with signals of any one direction will be one-sided to some extent.

Figure 9 is a fusion signal spectrum of $x$ and $y$ direction. Figure 9(a) is full vector spectrum; Figure 9(b) is the shaft orbit of the signal at different order. The synthetic signal of $X$ and $Y$ transducer at each harmonic is a motion ellipse, as shown in Figure 9(b). The major axis of the ellipse reflects the most significant vibration. No matter where the transducer mounts, the main vibration vector is the maximum amplitude of the vibration in the motion plane. On the same rotor section, the main vibration vector is not affected by the radial mounting position of the two transducers installed vertically with each other. The full vector spectrum is the synthesis of two homologous transducers' information. It works as a virtual transducer in the plane to detect the maximum vibration of the interface at each frequency. So its spectral structure can reflect the operation state of the machine comprehensively. The vibration amplitude distribution of the full vector spectrum is in accordance with shaft orbit; Figure 9 shows that the vibration amplitude mainly relates to the double frequency, and the actual situation is also the case.

4.2. Prediction of Full Vector Amplitude. The fault severity can be determined by analyzing the vibration amplitude. Full vector data fusion was carried out based on the collected vibration signals to extract the full vector amplitude of rotating frequency. The $\mathrm{AR}(4)$ data prediction model was established. The time interval is 2 hours. The method is suitable for the long-term monitoring and numerical prediction of stable operating machines. Figure 10 indicates that the predicted value is almost the same as the measured vibration value. The established AR prediction model of full vector information fusion could predict the change of vibration effectively. By using full vector amplitude, the one-sidedness of one single transducer can be overcome.

4.3. Prediction of Full Vector Spectrum. The fault position and property can be determined in accordance with the machine structure by analysis the spectrum structure [28]. To predict the fault property, spectrum structure prediction is a feasible method.

Obtaining signal with one single transducer, whether the transducer mounts in $x$ direction or in $y$ direction, the acquired signal is one-sided for fault prediction. The reliability of conclusion concerning fault diagnosis and fault character prediction with such signals cannot be guaranteed. Date fusion must be carried out to enhance the reliability of spectrum. The data fusion is based on the theory of full vector spectrum, and the main vector was used for the prediction of spectrum structure. The main vector sequence reflects the distribution of the vibration energy along the frequency. Compared with the spectrum in one direction only, the full vector spectral structure can reflect the operation state of the machine comprehensively. The result of spectrum structure prediction with $\mathrm{AR}(13)$ is shown in Figure 11. The potential fault property can be determined by analyzing the spectrum structure in the prediction domain and reasonable maintenance can be arranged.

4.4. Equipment Maintenance Using Predicted Feature. Vibration analysis is one of the important methods for fault diagnosis of rotating machinery. By collecting the vibration signal and extracting the eigenvector, the established vibration analysis and prediction system can be used as an important support for equipment health management. By analyzing and predicting the vibration feature, it is possible to evaluate the operating status of the equipment, predict deterioration trends, and identify potential failures that may occur.

The established AR prediction model can predict the changes of vibration amplitude and full vector spectrum. By predicting the change of the amplitude, it is possible to determine the deterioration tendency of the equipment and to predict how long the alarm will occur. When the alarm is predicted to occur in the near future, the full vector 


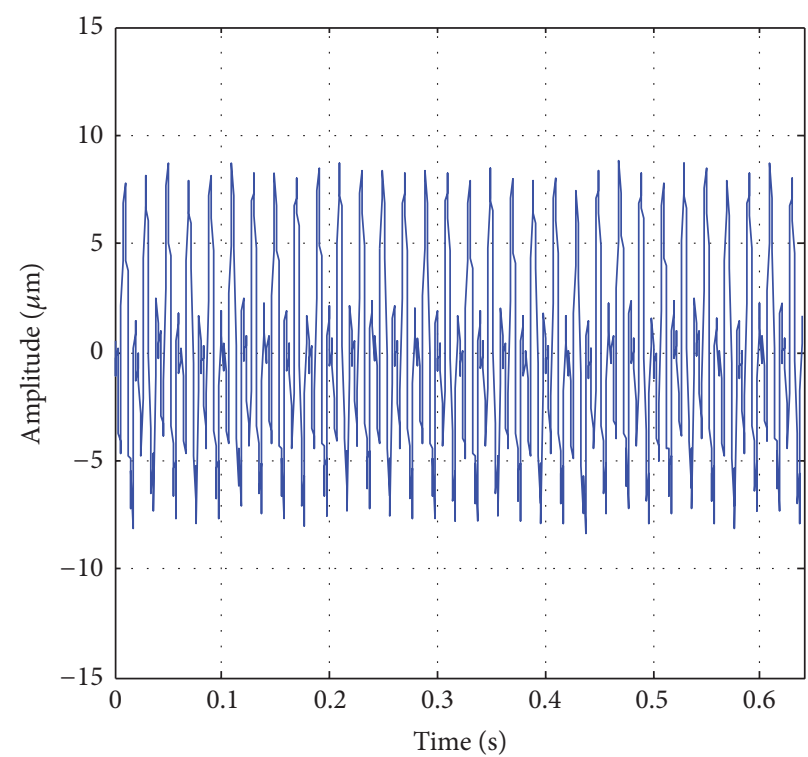

(a) Wave of $x$-direction

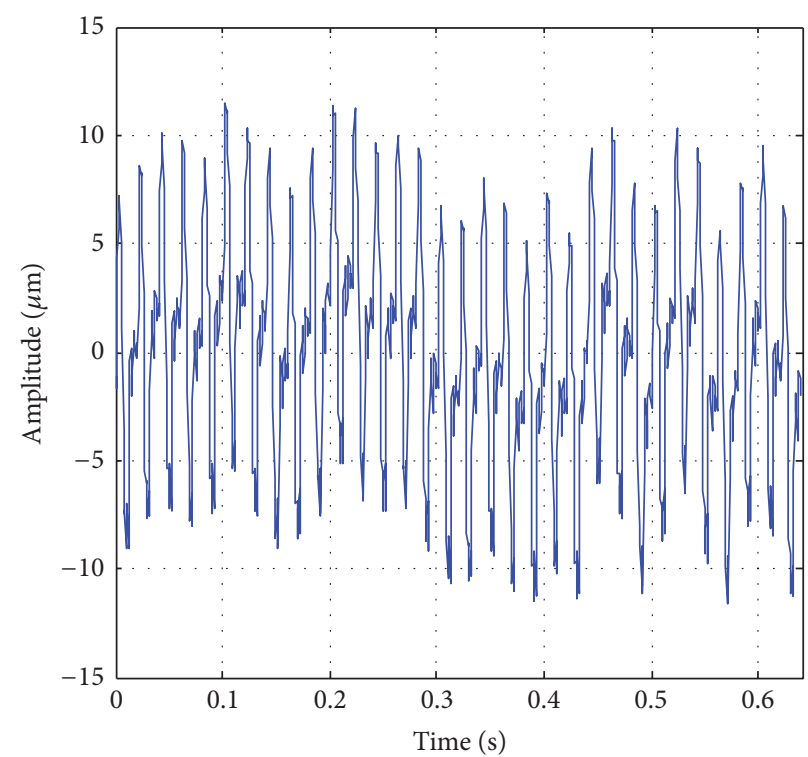

(b) Wave of $y$-direction

FIGURE 7: Vibration wave of $x$ and $y$ direction of the same cross section.

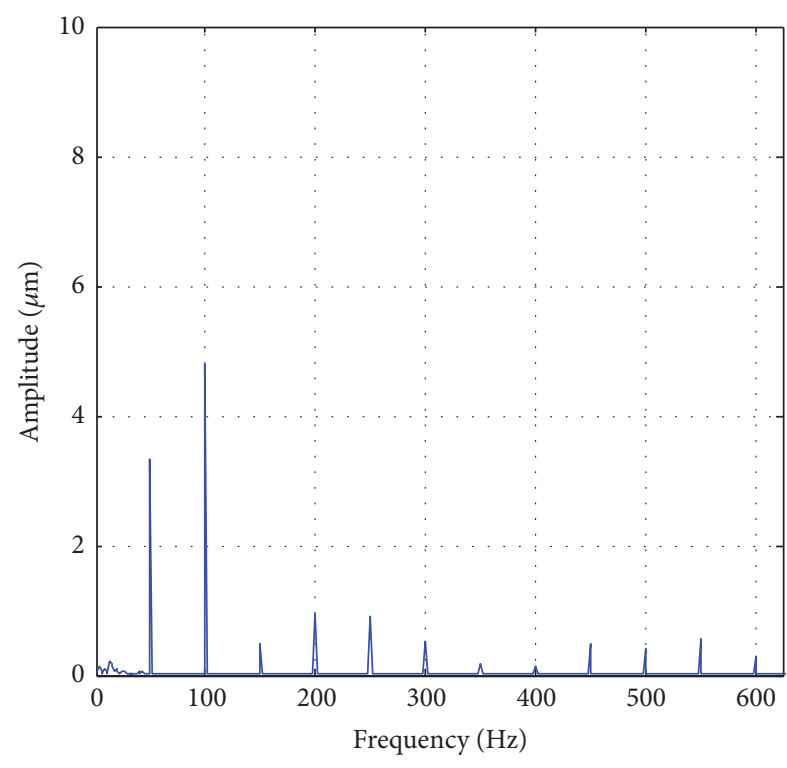

(a) Spectrum of $x$-direction

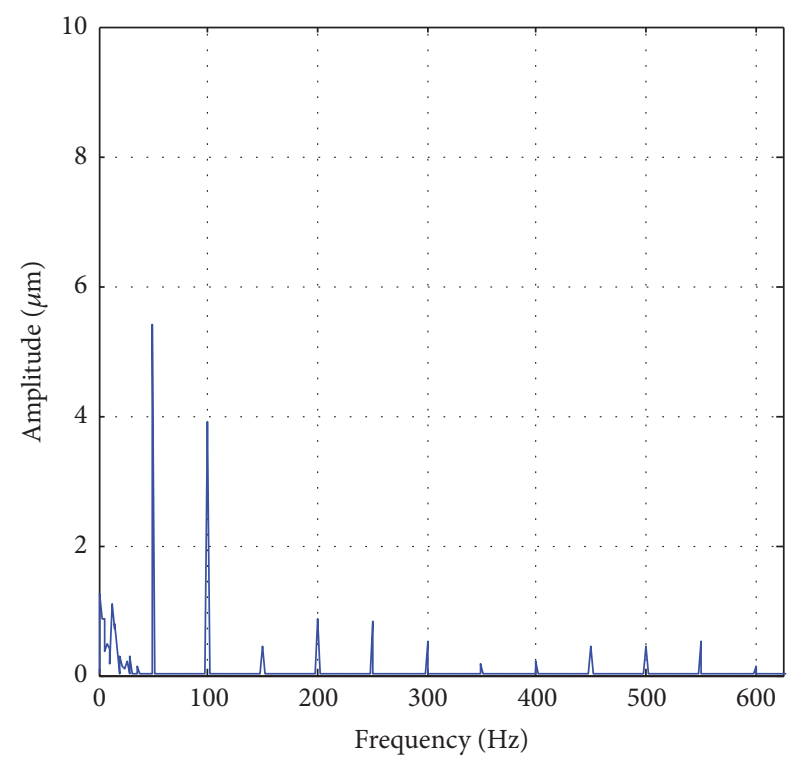

(b) Spectrum of $y$-direction

Figure 8: Spectrum of $x$ and $y$ direction of the same cross section.

spectrum prediction model is called to check the amplitude changes at different characteristic frequencies. The characteristic frequency is related to the corresponding equipment failure; for example, rotating frequency is related to the failure of dynamic unbalance. By examining the changes of the amplitude of the characteristic frequency in the spectrum, it is possible to judge out the possible fault to occur. The vibration feature prediction can provide a decision-making basis for the equipment management and maintenance department.

4.5. Discussions. In the machine condition monitoring system, the AR model is an effective prediction method. It can be used to predict the change value of the vibration. The structure of the prediction model can be determined by the order number of the AR model. The order of the model was fixed. The coefficients of each order can be determined by the calculation of the sample data. In the actual condition monitoring system, the sampling data was changed as time goes on. The coefficients of each order can be modified to fit in with the latest running state by calculating the sample data in the latest period of time.

For the data feature extraction, since the full vector amplitude can reflect the maximum vibration amplitude of the equipment, the prediction model was constructed based 


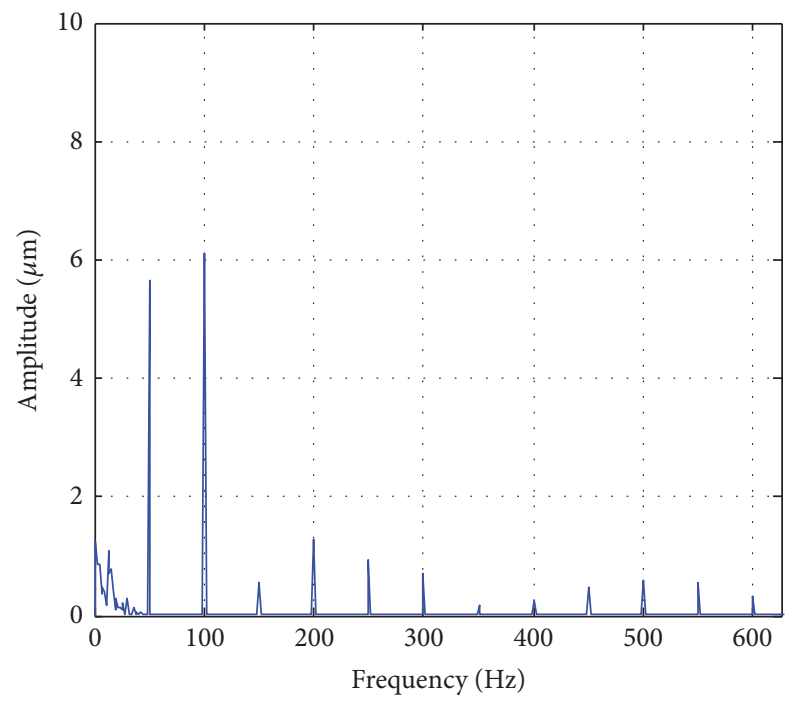

(a) Full vector spectrum

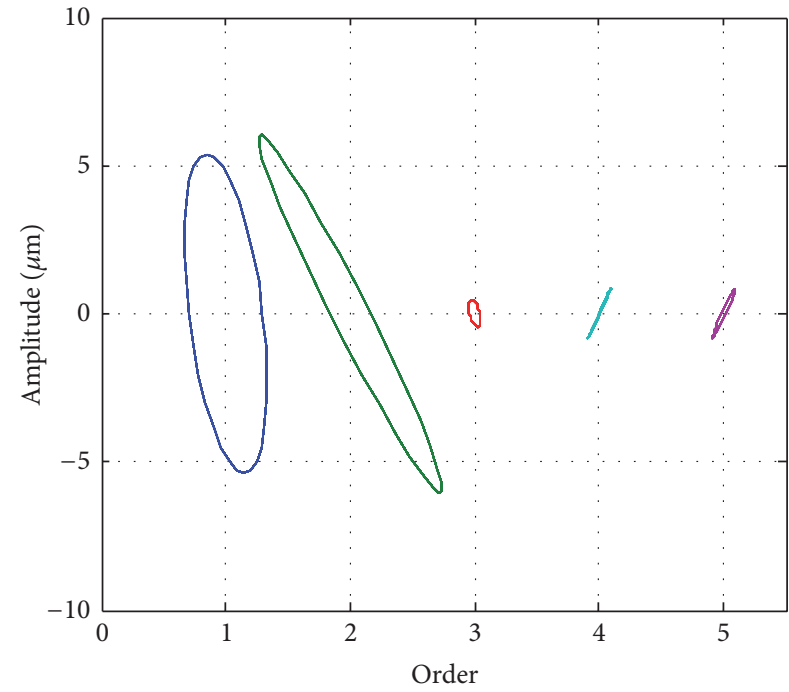

(b) Shaft orbit

FIGURE 9: Full vector spectrum and shaft orbit.

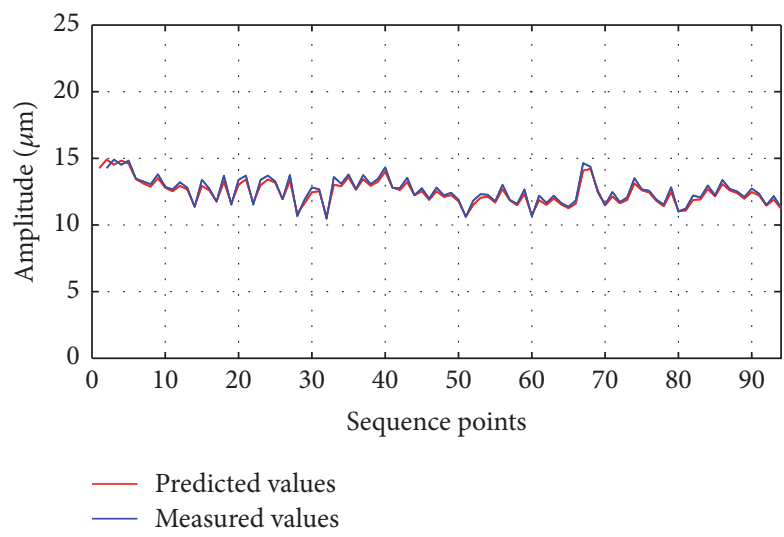

FIGURE 10: Comparison between predicted and measured values.

on the full vector amplitude. The AR amplitude prediction model can forecast the change trend of the equipment effectively. By analyzing the structure characteristics and the change of full vector spectrum, the fault property and failure position can be predicted. Compared with signals with a single transducer, the full vector spectrum is more effective to reflect the fault condition of the equipment.

\section{Conclusions}

Signal feature extraction and prediction model establishment were two important aspects in the process of the fault prediction. The full vector fusion and fault prediction were studied with the turbine as the research object. The construction of the AR prediction model can effectively solve the problem of vibration data prediction. Since the signal of a single transducer has shortage of one-sidedness for the machine fault diagnosis, the information fusion was

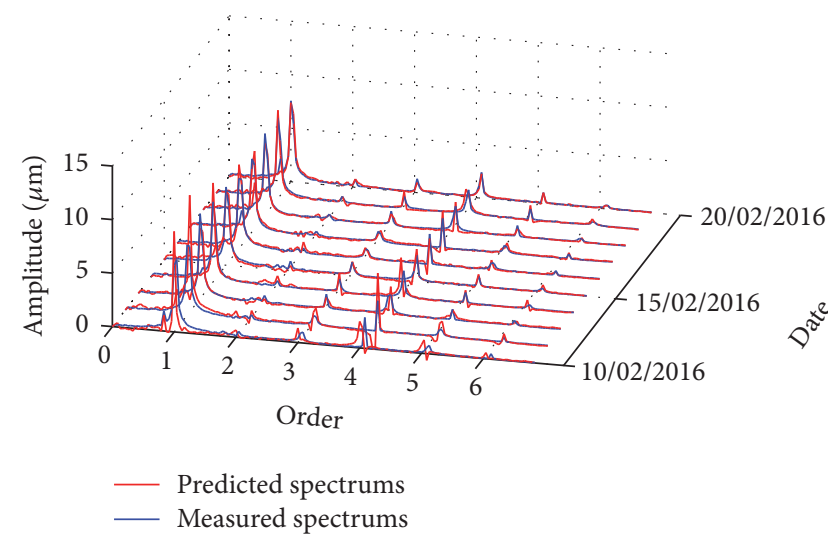

FIGURE 11: Full vector spectrum prediction with AR(13).

accomplished based on the theory of full vector spectrum. The full vector amplitude was extracted to reflect the strength of the equipment. The full vector spectrum was figured out to analyze the fault property. The amplitude and spectrum of fused signal can overcome the shortage of one-sidedness.

The established AR prediction model based on the time series full vector amplitude can determine the change of vibration intensity effectively. The established AR spectrum prediction model based on the time series full vector spectrum can predict the change of the structure of the vibration spectrum effectively. According to the failure mechanism of the machine equipment, it is possible to determine the nature and location of the fault by analyzing the predicted frequency spectrum. The study on signal feature extraction based on the theory of full vector spectrum and the construction of prediction model based on the theory of AR model lays a technical foundation for the further research of prognostic and health management. 


\section{Conflicts of Interest}

The authors declare that there are no conflicts of interest regarding the publication of this paper.

\section{Acknowledgments}

This project is supported by the Guidance Program of the Key Projects of Scientific and Technical Research of Department of Education of Henan Province (13B603970.0), Natural Science Research Program of Department of Education of Henan Province (2010B460015), and the Opening Project of Key Laboratory of Precision Manufacturing Technology and Engineering, Henan Polytechnic University (PMTE201302A).

\section{References}

[1] J. Han and L. D. Shi, Full Vector Spectrum Technology and Its Engineering Application, China Machine Press, Beijing, China, 2008.

[2] L. Chen, J. Han, W. Lei, Y. Cui, and Z. Guan, "Full-vector signal acquisition and information fusion for the fault prediction," International Journal of Rotating Machinery, vol. 2016, Article ID 5980802, 7 pages, 2016.

[3] J. Ma, X. L. Xu, and D. H. Zhou, "Survey of fault prediction methods for rotating machinery," Process Automation Instrumentation, vol. 32, no. 8, pp. 1-3, 2011.

[4] S. Su, W. Zhang, and S. Zhao, "Fault prediction for nonlinear system using sliding ARMA combined with online LS-SVR," Mathematical Problems in Engineering, vol. 2014, Article ID 692848, 9 pages, 2014.

[5] Z. Zhang, Y. Wang, and K. Wang, "Intelligent fault diagnosis and prognosis approach for rotating machinery integrating wavelet transform, principal component analysis, and artificial neural networks," International Journal of Advanced Manufacturing Technology, vol. 68, no. 1-4, pp. 763-773, 2013.

[6] K. Wang, J. Deng, and Y. Yang, "A fault prediction method of rotating machinery based on an improved empirical mode decomposition," Advanced Science Letters, vol. 5, no. 2, pp. 874877, 2012.

[7] A. K. Verma, S. Sarangi, and M. Kolekar, "Misalignment Fault Prediction of Motor-Shaft Using Multiscale Entropy and Support Vector Machine," Advances in Intelligent Systems and Computing, vol. 320, pp. 359-370, 2015.

[8] S. Kiakojoori and K. Khorasani, "Dynamic neural networks for gas turbine engine degradation prediction, health monitoring and prognosis," Neural Computing and Applications, vol. 27, no. 8, pp. 2157-2192, 2016

[9] P. Kesaba, B. B. Choudhury, and M. K. Muni, "An Effective prediction of position analysis of industrial robot using fuzzy logic approach," in Proceedings of $3 r d$ International Conference on Advanced Computing, Networking and Informatics, vol. 43 of Smart Innovation, Systems and Technologies, pp. 45-54, Springer, New Delhi, India, 2016.

[10] S. K. Yang and T. S. Liu, "State estimation for predictive maintenance using Kalman filter," Reliability Engineering and System Safety, vol. 66, no. 1, pp. 29-39, 1999.

[11] A. Ray and S. Tangirala, "Stochastic modeling of fatigue crack dynamics for on-line failure prognostics," IEEE Transactions on Control Systems Technology, vol. 4, no. 4, pp. 443-451, 1996.
[12] G. Li, S. J. Qin, Y. Ji, and D. Zhou, "Reconstruction based fault prognosis for continuous processes," Control Engineering Practice, vol. 18, no. 10, pp. 1211-1219, 2010.

[13] R. C. M. Yam, P. W. Tse, L. Li, and P. Tu, "Intelligent predictive decision support system for condition-based maintenance," International Journal of Advanced Manufacturing Technology, vol. 17, no. 5, pp. 383-391, 2001.

[14] B. Samanta and C. Nataraj, "Prognostics of machine condition using energy based monitoring index and computational intelligence," in Proceedings of the ASME International Design Engineering Technical Conferences and Computers and Information in Engineering Conference (IDETC/CIE '08), vol. 3, pp. 13471358, Brooklyn, NY, USA, August 2008.

[15] L.-H. Lin, J. Ma, and X.-L. Xu, "The turbine machine fault prediction based on kernel principal component analysis," Advanced Materials Research, vol. 383-390, pp. 4787-4791, 2012.

[16] A. Verma, S. Sarangi, and M. H. Kolekar, "Stator winding fault prediction of induction motors using multiscale entropy and grey fuzzy optimization methods," Computers and Electrical Engineering, vol. 40, no. 7, pp. 2246-2258, 2014.

[17] D. E. Bently, C. T. Hatch, and B. Grissom, Fundamentals of Rotating Machinery Diagnostics, Bently Pressurized Bearing Press, Minden Nev, USA, 2002.

[18] L. S. Qu, Holospectrum and Holobalancing Technique in Machinery Diagnosis, Beijing Science Press, Beijing, China, 2007.

[19] A. Muszynska, Rotordynamics, Taylor Francis Group, New York, NY, USA, 2005.

[20] J. P. Nolan and N. Ravishanker, "Simultaneous prediction intervals for ARMA processes with stable innovations," Journal of Forecasting, vol. 28, no. 3, pp. 235-246, 2009.

[21] D. H. Yi, Time Series Analysis Method and Application, China Renmin University Press, Beijing, China, 2011.

[22] Y. Lu and F. Gao, "A novel time-domain auto-regressive model for structural damage diagnosis," Journal of Sound and Vibration, vol. 283, no. 3-5, pp. 1031-1049, 2005.

[23] H. Akaike, "A Bayesian analysis of the minimum AIC procedure," Annals of the Institute of Statistical Mathematics, vol. 30, no. 1, pp. 9-14, 1978.

[24] H. Akaike, "Maximum likelihood identification of Gaussian autoregressive moving average models," Biometrika, vol. 60, pp. 255-265, 1973.

[25] A. Spanos, "Akaike-type criteria and the reliability of inference: model selection versus statistical model specification," Journal of Econometrics, vol. 158, no. 2, pp. 204-220, 2010.

[26] M. Piłatowska, "Information and prediction criteria in selecting the forecasting model," Dynamic Econometric Models, vol. 11, pp. 21-40, 2011.

[27] G. X. Wu, "Comment and improvement on criteria of order determination of sequence model," Journal of Xian Mining Institute, vol. 15, no. 4, pp. 307-309, 1995.

[28] J. M. Vance, Rotordynamics of Turbomachinery, WileyInterscience, New York, NY, USA, 1987. 


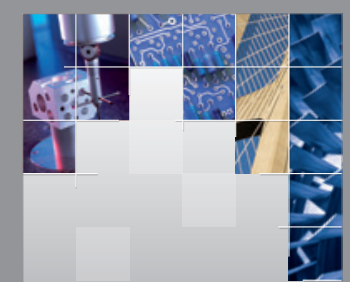

\section{Enfincering}
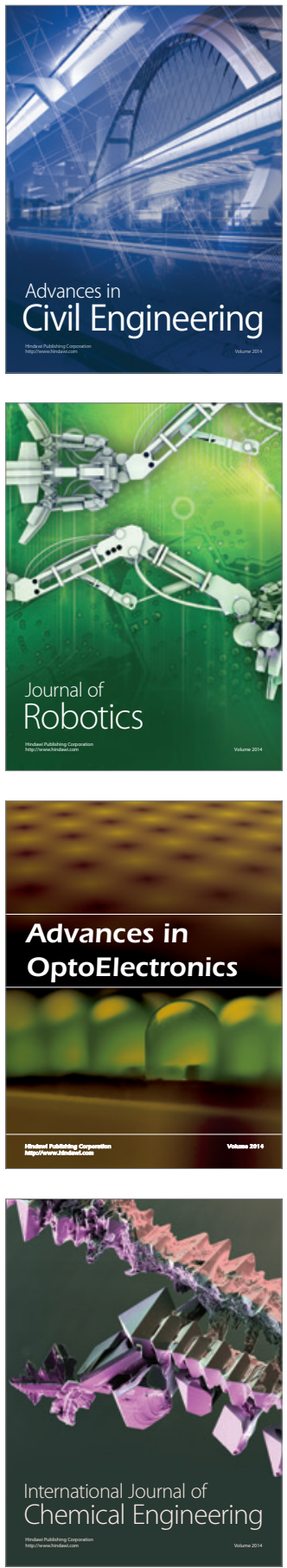

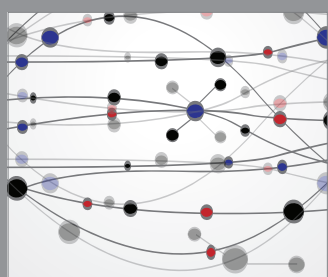

The Scientific World Journal

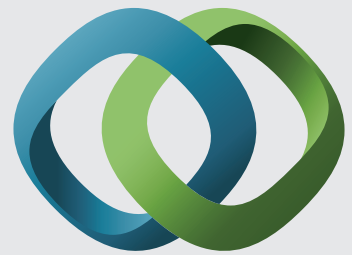

\section{Hindawi}

Submit your manuscripts at

https://www.hindawi.com
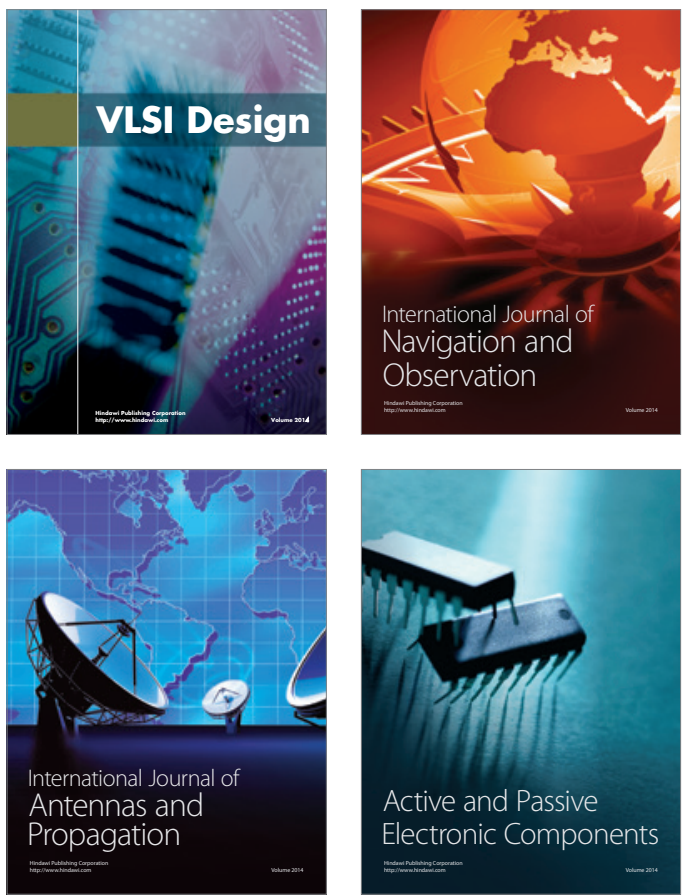
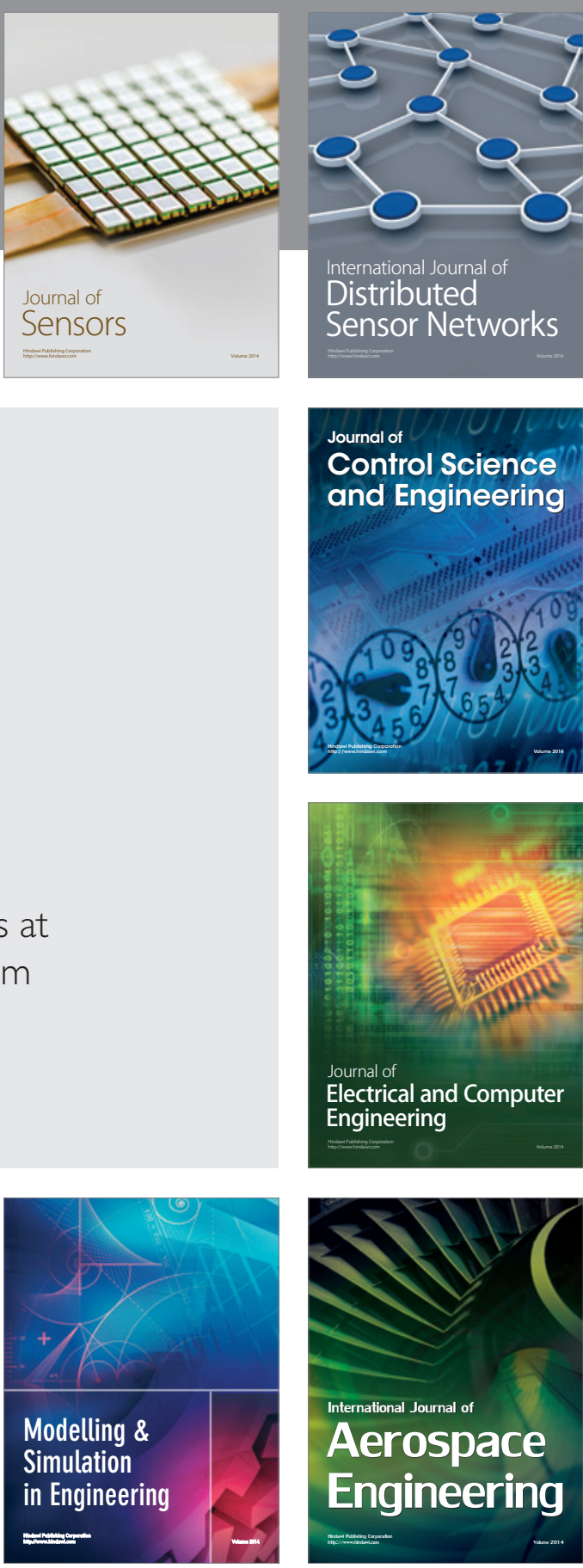

International Journal of

Distributed

Sensor Networks

$-$

Joumal of

Control Science

and Engineering
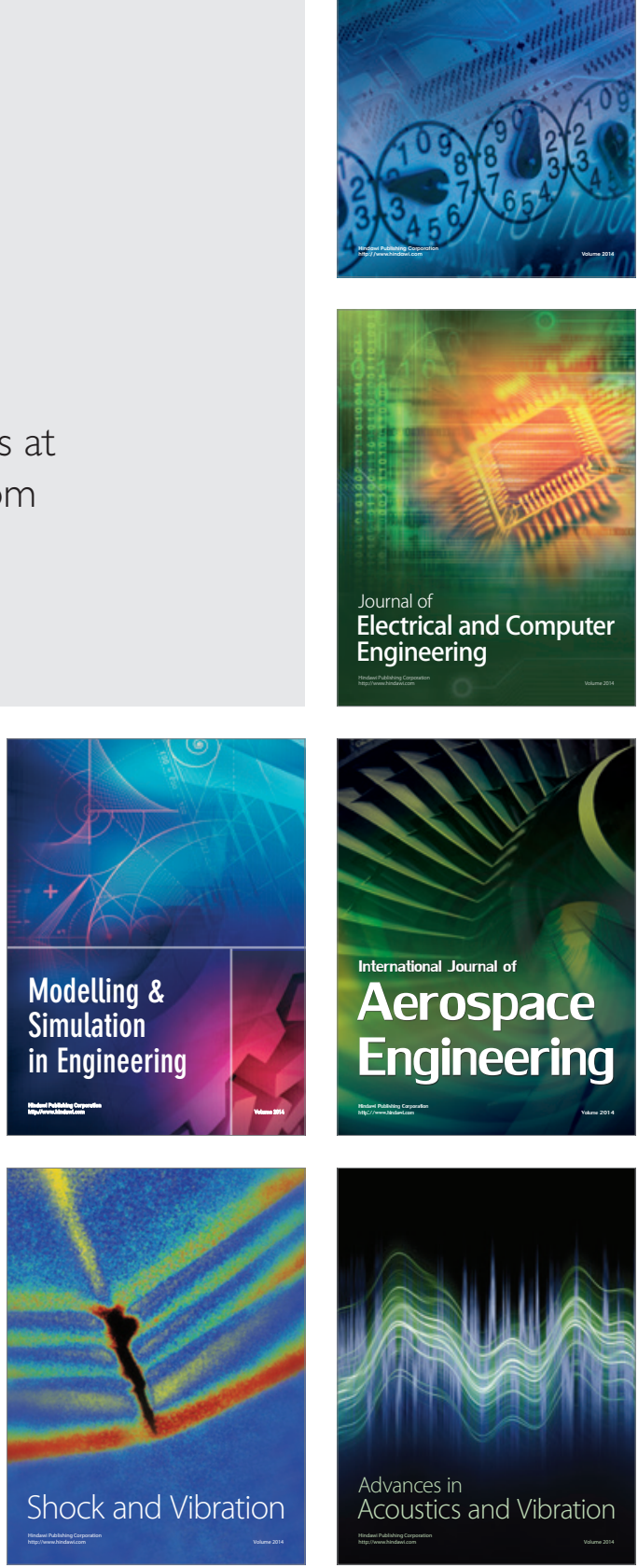